\title{
The Use of M2-Pyruvate Kinase as a Stool Biomarker for Detection of Colorectal Cancer in Tertiary Teaching Hospital: A Comparative Study
}

\author{
Shahidah Che Alhadi ${ }^{1, \star}$, Wan Zainira Wan Zain ${ }^{2,3, \star}$, Zalina Zahari ${ }^{4}$, Mohd Nizam Md Hashim³, \\ Syed Hassan Syed Abd. Aziz ${ }^{2,3}$, Zaidi Zakaria ${ }^{2,3}$, Michael Pak-Kai Wong ${ }^{2,3}$, Andee Dzulkarnaen Zakaria ${ }^{2,3}$ \\ ${ }^{1}$ Department of Surgery, Kulliyyah of Medicine, International Islamic University Malaysia, Kuantan; ${ }^{2}$ Department of Surgery, School of \\ Medical Sciences, Universiti Sains Malaysia, Kubang Kerian; ${ }^{3}$ Department of Surgery, Hospital Universiti Sains Malaysia, Kubang Kerian; \\ ${ }^{4}$ Faculty of Pharmacy, Universiti Sultan Zainal Abidin, Besut Campus, Besut, Malaysia
}

Purpose: Guaiac fecal occult blood test (gFOBT) has been the standard for colorectal screening but it has low sensitivity and specificity. This study evaluated the use of fecal tumor M2-pyruvate kinase (M2-PK) for detection of colorectal cancer and to compare with the current surveillance tool; gFOBT in symptomatic adult subjects underwent colonoscopy.

Methods: Stool samples were collected prospectively from symptomatic adults who had elective colonoscopy from September 2014 to January 2016 and were analyzed with the ScheBo M2-PK Quick test and laboratory detection of fecal hemoglobin.

Results: The results were correlated to the colonoscopy findings and/or histopathology report. Eighty-five subjects (age of $56.8 \pm 15.3$ years [mean \pm standard deviation]) were recruited with a total of 17 colorectal cancer $(20.0 \%)$ and 10 colorectal adenoma patients (11.8\%). The sensitivity of M2-PK test in colorectal cancer detection was higher than gFOBT (100\% vs. 64.7\%). M2-PK test had a lower specificity when compared to gFOBT (72.5\% vs. $88.2 \%)$ in colorectal cancer detection. The positive and negative predictive values were $47.2 \%$ and $100 \%$ for M2-PK test and 57.9\% and $90.9 \%$ for gFOBT. Conclusion: Fecal M2-PK Quick test has a high sensitivity for detection of colorectal cancer when compared to gFOBT, making it the potential choice for colorectal tumor screening biomarker in the future.

Keywords: Tumor biomarkers; Colorectal neoplasms; Occult blood; Pyruvate kinase type M2

Received: May 15, 2020 - Revised: Aug 9, 2020 - Accepted: Aug 27, 2020 Correspondence to: Wan Zainira Wan Zain, MMed (GenSurg) Department of Surgery, School of Medical Sciences, Universiti Sains Malaysia, 16150 Kubang Kerian, Kelantan, Malaysia

Tel: +60-9-7676774, Fax: + 60-9-7653370

E-mail: zainira@usm.my

ORCID: https://orcid.org/0000-0001-8019-6063

Co-Correspondence to: Zalina Zahari, Ph.D.

Faculty of Pharmacy, Universiti Sultan Zainal Abidin, Besut Campus, 22200 Besut, Terengganu, Malaysia

Tel: +60-9-6993187, Fax: +60-9-6993004

E-mail: zalinazahari@unisza.edu.my

ORCID: https://orcid.org/0000-0003-1459-8958

*Shahidah Che Alhadi and Wan Zainira Wan Zain contributed equally to this study as co-first authors.

(C) 2020 The Korean Society of Coloproctology

This is an open-access article distributed under the terms of the Creative Commons Attribution NonCommercial License (https://creativecommons.org/licenses/by-nc/4.0) which permits unrestricted noncommercial use, distribution, and reproduction in any medium, provided the original work is properly cited.

\section{INTRODUCTION}

According to World Health Organization's GLOBOCAN 2012, it was estimated about 37,400 persons were diagnosed with new cancer in Malaysia and colorectal cancer is the second most common cancer in men (14.1\%) and the third in women (10.2\%) [1]. The incidence and mortality of colorectal cancer are rising rapidly in Asia [2], despite the availability of screening methods.

Colorectal cancer can be prevented by detection of early-stage adenocarcinoma, and detection and removal of adenomatous polyps. Incidence and mortality due to colorectal cancer can be effectively reduced with early diagnosis, if regular screening is done [3]. However, Yusoff et al. [4] showed that participation in colorectal cancer screening among Malaysians was extremely low mainly due to embarrassment, uncomfortable and unsure the screening test availability. 
Current gold standard screening tool for early detection of colorectal cancer and high-risk adenoma is colonoscopy, which has the highest sensitivity and specificity. However, most patients refused to participate because of its invasiveness. Colonoscopy is associated with bleeding, perforations, and cardiopulmonary complication from drugs used for sedation [5]. Fecal test is much more acceptable as it is noninvasive $[4,6]$. Currently, available stool tests for colorectal cancer screening are fecal occult blood test (FOBT), genetic stool test, and M2-pyruvate kinase (M2-PK) stool test. The most widely used is FOBT [7]. According to Malaysian Clinical Practice Guidelines on colorectal cancer screening 2001, the best approach to screen for colorectal cancer is by using FOBT as an annual screening to those at average risk [8]. However, FOBT is limited by its low sensitivity toward colorectal polyps and frequent false-positive results [9]. Stool DNA test is not recommended by the Asia Pacific Consensus on colorectal cancer as this test is still in experimental phase and is not ready to be used as a routine screening test [10].

Pyruvate kinase is an enzyme that is involved in the glycolysis process. Type M2-PK is a special isoenzyme of pyruvate kinase, which is expressed by proliferating cells $[11,12]$. The dimeric form termed tumor M2-PK is predominantly overexpressed in tumor cells $[11,13,14]$. M2-PK is essential in tumor growth of colorectal cancer by regulating cell proliferation and migration of colon cancer cells. A greater amount of this isomer is found at the advanced tumor stage $[12,15]$ and associated with lymph metastasis in colorectal cancer patients [12]. Tumor M2-PK has also been found in the stool of patients with colorectal cancer [10] and colorectal polyps [11]. The amount of M2-PK in stool can be quantified by enzyme-linked immunosorbent assay (ELISA) [11] and thus, fecal M2-PK has been proposed as a tool for detecting colorectal cancer [16-18].

The aim of this study was to evaluate the use of fecal tumor M2PK in detection of colorectal cancer in symptomatic adult subjects who underwent colonoscopy. The specific objectives of the study were to determine the sensitivity, specificity, positive predictive value, and negative predictive value of fecal M2-PK test toward detection of colorectal cancer and to compare the diagnostic accuracy of fecal M2-PK with the current surveillance tool; guaiac FOBT (gFOBT) in detecting colorectal cancer.

\section{METHODS}

\section{Study design and setting}

This prospective study was carried out on patients that underwent colonoscopy for various indications at Hospital Universiti Sains Malaysia (HUSM) from September 2014 until January 2016.

\section{Participants}

All subjects were evaluated and screened for study eligibility prior to study entry. This was a convenience sample of patients that underwent colonoscopy for reasons such as colorectal cancer screen- ing, investigation of colonic symptoms, high risk for colorectal cancer, family history of colorectal neoplasia, and clinical suspicion of colorectal cancer. Patients that had past history of colectomy, patients known to have colorectal cancer and came for surveillance colonoscopy, underwent chemotherapy for colorectal cancer, or incomplete colonic examination were excluded from the study.

The sample size was calculated for diagnostic accuracy study based on the reported sensitivity of a positive fecal tumor M2-PK test for colorectal cancer of $97 \%$ and specificity of $98 \%$ [19]. The estimated sample size for this study was 85 samples.

\section{Stool collection, FOBT, M2-PK testing, and colonoscopy}

All patients included in this study were given information leaflets and explained the procedures and study objectives. The written informed consents were obtained from the patients.

Two stool samples were collected from each patient before bowel preparation for colonoscopy was started. No dietary restrictions were required prior to stool collections. For gFOBT, the stool sample was sent to HUSM's laboratory for detection of hemoglobin in the stool. For M2-PK test, the stool sample was tested using a commercially available rapid test, ScheBo M2-PK Quick (ScheBo Biotech AG, Giessen, Germany) according to the manufacturer's instruction. A single trained person performed the stool testing for M2-PK.

Colonoscopy was performed by experienced endoscopists according to the current practice at Endoscopy Unit of HUSM, with visualization of the entire colon up to caecum using a standard colonoscopy after routine bowel preparation. Patients with incomplete colonoscopy proceeded with computed tomography (CT) colonography or barium enema as to complete their colonic examination. All lesions found during colonoscopy were biopsied and the tissue biopsies were sent to the Pathology Department of HUSM for histopathology processing. Pathologists were not aware of the gFOBT and M2-PK test results. The final diagnosis of each patient was based on the combined results of colonoscopy and histopathology examination. The results of M2-PK test and gFOBT were labeled as positive or negative and both were analyzed and compared to the colonoscopic findings and histopathology examination.

\section{Ethical considerations}

Ethical clearance was obtained from the Human Research Ethics Committee, Universiti Sains Malaysia in Kelantan, Malaysia (No. USM/JEPeM/1404166).

\section{Data analysis}

Cross tabulation was done to determine the true-positive, truenegative, false-positive, and false-negative results. Calculation for diagnostic test evaluation, which includes sensitivity, specificity, positive predictive value, negative predictive value, positive likelihood ratio, and negative likelihood ratio were done using statisti- 
cal program MedCalc, ver. 16.2.1 (MedCalc Software Ltd., Ostend, Belgium). The discriminant accuracy of each marker was estimated as the area under the receiver-opening characteristics (ROC) curve. Statistical analysis was carried out using IBM SPSS Statistics for Windows (ver. 22, IBM Corp., Armonk, NY, USA). The limit of significance was set at 0.05 .

\section{RESULTS}

\section{Patient demographic and clinical characteristics}

Of the 138 subjects scheduled for colonoscopy, 94 met the inclusion and exclusion criteria and enrolled in this study. Eighty-five subjects were included and 9 subjects were excluded. Table 1 shows the characteristics of the study population. All 94 subjects completed fecal tumor M2-PK test and FOBT. Among the subjects, 69 participants completed colonoscopy. Five subjects had further colonic examination by CT colonography $(n=3)$ or barium enema $(n=2)$. Another 11 subjects had obstructed colonic tumor and were included in this study. The remaining 9 subjects were excluded due to incomplete colonic examination without further imaging. A total of 17 colorectal cancers (20.0\%) and 10 colorectal adenomas (11.8\%) were detected in 85 colonoscopies. The remaining 58 patients (68.2\%) had normal or nonneoplastic disease. Of the 17 patients with colorectal cancers, 11 patients had incomplete colonic examination due to obstructed tumor. Fig. 1 shows the flow of participants through the study.

\section{Test characteristics in relation to colonoscopy findings}

Table 2 summarizes the test characteristic in relation to colonoscopy findings. M2-PK tests were positive in all 17 patients diagnosed with colorectal cancer, 2 out of 10 patients (20.0\%) with

Table 1. Patient demographic and clinical characteristics

\begin{tabular}{lc}
\hline Characteristic & Data \\
\hline Age (yr) & $56.8 \pm 15.3$ \\
Sex & \\
$\quad$ Male & $50(58.8)$ \\
Female & $35(41.2)$ \\
Ethnicity & \\
Malay & $75(88.2)$ \\
Chinese & $9(10.6)$ \\
Indian & $1(1.2)$ \\
Indications for colonoscopic examination & \\
Altered bowel habit & $51(60.0)$ \\
Gastrointestinal bleeding & $17(20.0)$ \\
Abdominal pain & $5(5.9)$ \\
Abdominal mass & $2(2.4)$ \\
Others & $10(11.8)$ \\
\hline
\end{tabular}

Values are presented as mean \pm standard deviation or number (\%). colorectal adenoma, and 17 out of 58 patients (29.3\%) with normal colonoscopy. The gFOBT tests were positive in 11 out of $17 \mathrm{pa}-$ tients $(64.7 \%)$ with colorectal cancer, 3 out of 10 patients $(30.0 \%)$ with colorectal adenoma, and 5 out of 58 patients (8.6\%) with normal colonoscopy.

\section{Performance characteristics of fecal tumor M2-PK and gFOBT in detection of colorectal cancer and colorectal adenoma}

Table 3 depicts the performance characteristics of M2-PK against gFOBT in relation to colorectal cancer and colorectal adenoma.

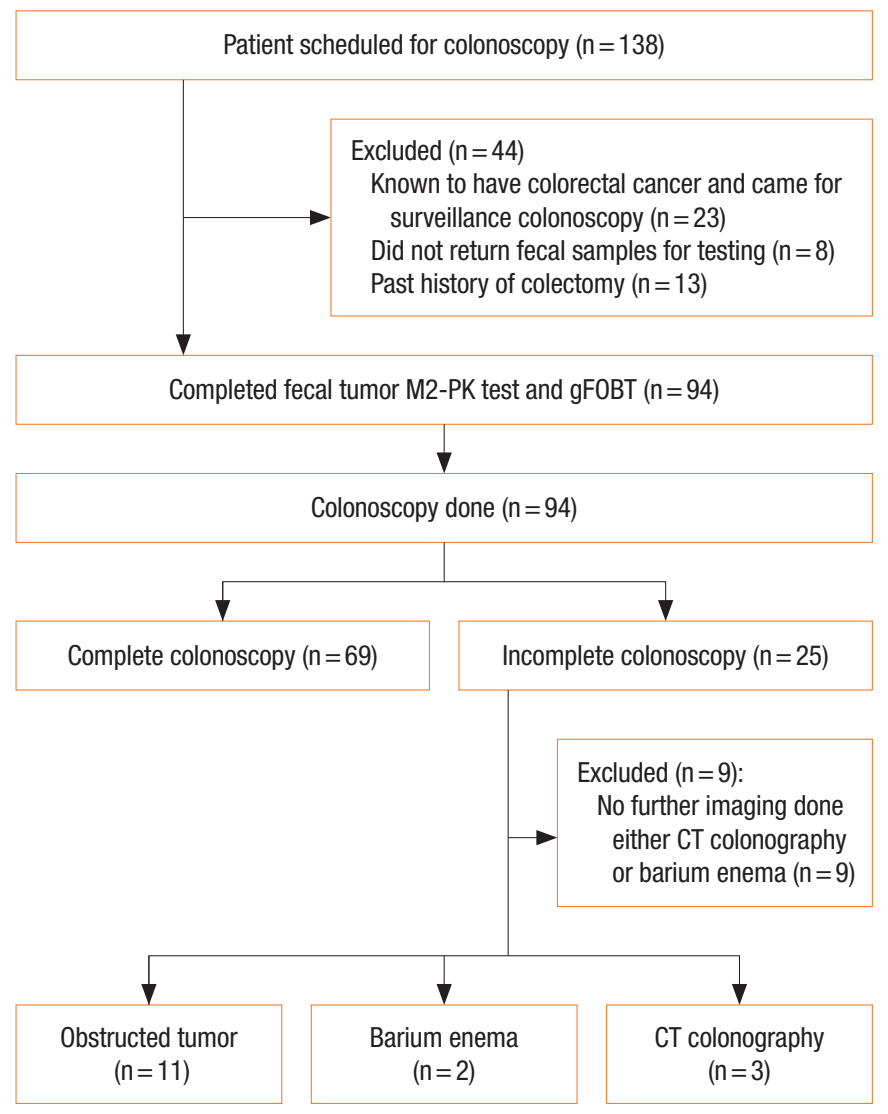

Fig. 1. Flowchart of participants for enrollment through the study. M2-PK, M2-pyruvate kinase; gFOBT, guaiac fecal occult blood test; CT, computed tomography.

Table 2. Test characteristics in relation to colonoscopy findings

\begin{tabular}{lccc}
\hline Colonoscopy finding & Total & $\begin{array}{c}\text { M2-PK test } \\
\text { positive }\end{array}$ & $\begin{array}{c}\text { gFOBT test } \\
\text { positive }\end{array}$ \\
\hline Colorectal cancer & 17 & $17(100)$ & $11(64.7)$ \\
Colorectal adenoma & 10 & $2(20.0)$ & $3(30.0)$ \\
Normal or nonneoplastic lesion & 58 & $17(29.3)$ & $5(8.6)$ \\
\hline
\end{tabular}

Values are presented as number only or number (\%).

M2-PK, M2-pyruvate kinase; gFOBT, guaiac fecal occult blood test. 
Table 3. Performance characteristics of fecal tumor M2-PK and gFOBT in detection of colorectal cancer and colorectal adenoma

\begin{tabular}{lcc}
\hline Parameter & M2-PK (\%) & gFOBT (\%) \\
\hline $\begin{array}{l}\text { Sensitivity } \\
\text { Colorectal cancer }\end{array}$ & $100(80.5-100)$ & $64.7(38.3-85.8)$ \\
$\quad$ Colorectal adenoma & $20.0(2.52-55.6)$ & $30.0(6.7-65.3)$ \\
$\begin{array}{l}\text { Specificity } \\
\text { Colorectal cancer }\end{array}$ & $72.5(60.4-82.5)$ & $88.2(78.1-94.8)$ \\
Colorectal adenoma & $54.5(42.8-66.2)$ & $78.7(67.6-87.3)$ \\
Positive predictive value & & \\
Colorectal cancer & $47.2(30.4-64.5)$ & $57.9(33.5-79.8)$ \\
Colorectal adenoma & $5.6(0.7-18.7)$ & $15.6(3.4-39.6)$ \\
Negative predictive value & & \\
Colorectal cancer & $100(92.9-100)$ & $90.9(81.3-96.6)$ \\
Colorectal adenoma & $83.7(70.3-92.7)$ & $89.4(79.4-95.6)$ \\
Positive likelihood ratio & & \\
Colorectal cancer & $3.6(2.5-5.3)$ & $5.5(2.6-11.5)$ \\
Colorectal adenoma & $0.4(0.1-1.6)$ & $1.4(0.5-4.0)$ \\
Negative likelihood ratio & & $0.4(0.2-0.8)$ \\
Colorectal cancer & 0 & $0.9(0.6-1.4)$ \\
Colorectal adenoma & $1.5(1.0-2.1)$ & \\
\hline
\end{tabular}

Values are presented as percentage ( $95 \%$ confidence interval). M2-PK, M2-pyruvate kinase; gFOBT, guaiac fecal occult blood test.

The sensitivity of the M2-PK test toward detection of colorectal cancer was higher than that of gFOBT (100\% vs. $64.7 \%)$. However, fecal M2-PK test had a lower specificity toward diagnosing colorectal cancer when compared with gFOBT ( $72.5 \%$ vs. $88.2 \%)$. The positive predictive value and negative predictive value of fecal M2-PK test were $47.2 \%$ and $100 \%$, respectively, whereas for gFOBT were $57.9 \%$ and $90.9 \%$, respectively. Both fecal M2-PK and gFOBT had low sensitivity toward detection of colorectal adenoma (20.0\% vs. $30.0 \%)$. The specificity of M2-PK test was lower than gFOBT (54.4\% vs. $78.7 \%$ ) in detecting colorectal adenoma.

\section{ROC curve for fecal tumor M2-PK and gFOBT in detection of colorectal cancer}

Fig. 2 shows the ROC curve for fecal M2-PK and gFOBT in detection of colorectal cancer. The curves were statistically significant for both tests, with the area beneath was greater than $50.0 \%$. The M2-PK test area under the ROC curve was 0.868 (95\% confidence interval [CI], 0.794 to $0.941 ; \mathrm{P}<0.005$ ), whereas the gFOBT test area under the ROC curve was 0.765 (95\% CI, 0.621 to 0.908 ; $\mathrm{P}<0.005)$.

\section{DISCUSSION}

Gold standard screening tool for early detection of colorectal can-

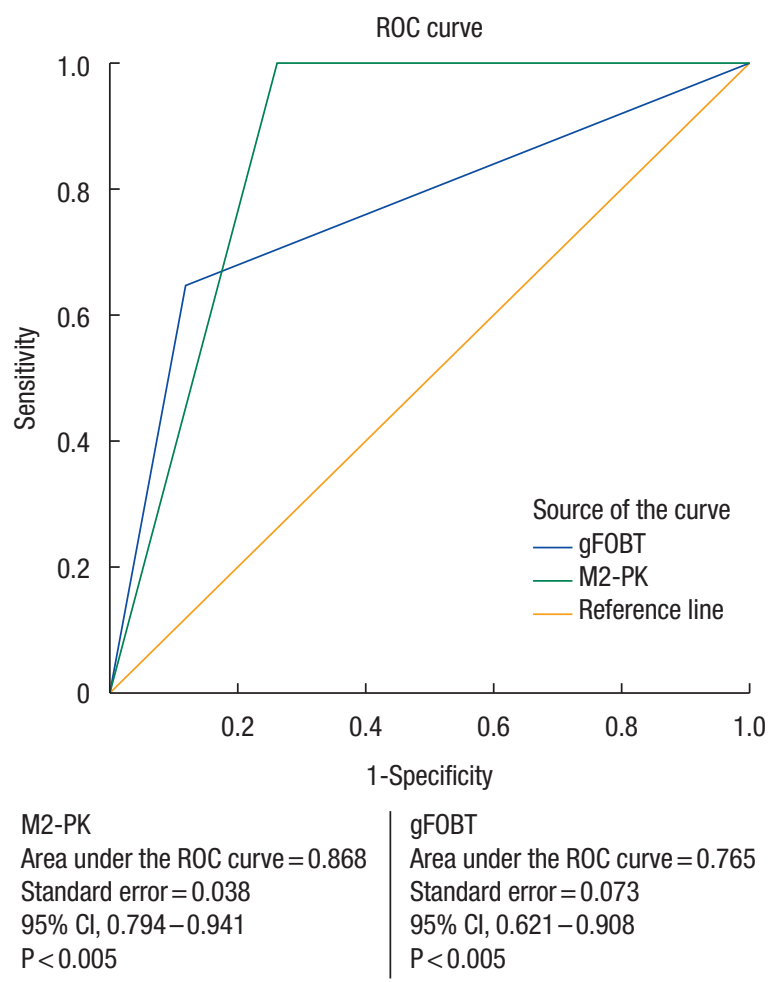

Fig. 2. Receiver-operating characteristics (ROC) curve for fecal tumor M2-pyruvate kinase (M2-PK) and guaiac fecal occult blood test (gFOBT) in detection of colorectal cancer. CI, confidence interval.

cer and high-risk adenoma is still colonoscopic examination with high sensitivity and specificity. However, the acceptance rates are very poor because of its inconvenience and invasiveness. A highly sensitive and specific yet noninvasive screening test for detection of colorectal cancer is needed to detect the possibility of colorectal cancer. Noninvasive tests such as fecal tests are much more acceptable as they are noninvasive and more comfortable. Current available stool tests for colorectal cancer screening are FOBT, genetic stool test, and M2-PK stool test.

In this study, the performance of fecal tumor M2-PK test for detection of colorectal cancer was evaluated and compared to gFOBT. The fecal M2-PK kit allowed the test to be done as soon as the stool sample is submitted by the patient and the result can be obtained immediately. Tumor M2-PK can be measured in feces of patients with colorectal cancer by using ELISA technique, favoring a potential tumor marker for colorectal cancer. The M2PK test is independent of occult blood (ELISA method), thus it can detect bleeding or nonbleeding gastrointestinal cancer and polyps [20].

A meta-analysis performed by Tonus et al. [20] reported relatively good performance of fecal tumor M2-PK in colorectal cancer screening with a mean sensitivity and specificity of $80.3 \%$ and 95.2\%, respectively. Recently, Sithambaram et al. [21] performed a case-control study on validation of the use of a rapid, point of care 
stool test M2-PK for the detection of colorectal cancer. The performance of fecal M2-PK Quick test revealed a sensitivity of $93.0 \%$, specificity of $97.5 \%$, and diagnostic accuracy of $96.0 \%$. The test was found to be highly accurate regardless of the tumor stages and location [21].

In our study, the sensitivity of the M2-PK test in detecting patients with colorectal cancer was $100 \%$, which was much superior to that of gFOBT (64.7\%). The published data on sensitivity ranged from $71.4 \%$ to $100 \%$ [14-16, 19, 21-23]. However, M2-PK test had slightly lower specificity than gFOBT $(72.5 \%$ vs. $88.2 \%)$. Our result for specificity is still within the published range of $71.0 \%$ to $98.0 \%$ [14-16, 19, 21-23].

This study showed that both fecal tumor M2-PK test and gFOBT area under the ROC curve for detection of colorectal cancer were statistically significant, with the area beneath was greater than $50.0 \%$, in which both tests were able to correctly classify those with and without colorectal cancer.

In our study, both fecal M2-PK test and gFOBT had low sensitivity in detecting colorectal adenoma. Correspondingly, a study by Haug et al. [24] found a low sensitivity of $22.0 \%$ for advanced adenoma and $23.0 \%$ for other adenomas, whereas specificity was $82.0 \%$. A meta-analysis by Tonus et al. [20] reported the sensitivity of $25.0 \%$ for adenoma $<1 \mathrm{~cm}$ in diameter, $44.0 \%$ for adenoma $>1 \mathrm{~cm}$, and $51.0 \%$ for adenoma of unspecified diameter. Therefore, due to its low sensitivity, fecal M2-PK has a very limited role in detection of colorectal adenoma.

Studies also showed that intestinal inflammation could cause elevation of fecal tumor M2-PK level. In a study by Shastri et al. [25], they reported that 185 of 276 patients with inflammatory bowel disease had a positive fecal M2-PK level (cut-off value, $>4$ $\mathrm{U} / \mathrm{mL}$ ). In another study by Abdullah et al. [16] found that fecal M2-PK test was positive in inflammatory bowel disease, infective colitis, and amebic colitis. Fecal M2-PK level was also found to be elevated in patients with pouchitis following ileal pouch-anal anastomosis for ulcerative colitis and familial adenomatous polyposis [26].

In a review by Kumar et al. [18], M2-PK level was found significantly higher in plasma of patients with other benign conditions, like bacterial infection, rheumatic diseases, acute and chronic pancreatitis, chronic cardiac failure, and diabetic nephropathy. In a large screening study, Haug et al. [24] observed a higher fecal M2-PK level in older age groups than in younger age groups. In another study by Haug et al. [22], there was an association reported between current smoking status and elevated tumor M2PK levels compared to never and former smokers. However, no association was noted between age, sex, body mass index, or family history of colorectal cancer and a positive fecal M2-PK test [22]. Our study did not evaluate the possibility of association between fecal M2-PK with patients' characteristics and medical illnesses that might cause false positivity of results. Although colonoscopy is the best tool for diagnosing colorectal cancer and highrisk adenoma, it can have a miss rate of $16.8 \%$ for colonic polyps,
$17.0 \%$ for adenomas, and 5.4\% for advanced adenoma [27]. Therefore, further study is needed to evaluate the association of these benign conditions with fecal M2-PK level for better understanding of its role in colorectal cancer screening.

There were some limitations found in this study. Our study investigated on symptomatic group going for colonoscopy. There may be overestimation of sensitivity and underestimation of specificity in hospital-based studies. Therefore, the results should be interpreted with caution when comparing with an average-risk population. For that reason, the application of fecal tumor M2-PK should be further studied in average-risk group and involved larger population in comparing fecal tumor M2-PK and gFOBT. Average-risk population are not included in this study as it is difficult to promote colonoscopy in this group as they did not have any risk except for their age. Although it is easy to get their stool sample, we need to confirm with colonoscopy with regards to their bowel examination. The recommended gFOBT testing is by using 3 stool specimens with dietary restrictions. However, in our study, the gFOBT was done only using single stool testing and without dietary restrictions. The sensitivity of gFOBT could differ if the testing was performed as recommendation.

In conclusion, the presence of M2-PK in stool warrant further investigation for diagnosis of colorectal cancer. Those with positive result of M2-PK test should undergo complete diagnostic evaluation. The features of fecal M2-PK test is highly sensitive, fast, and easy to be performed in clinical settings and is deemed suitable as a screening tool and complimentary test for colorectal tumor.

\section{CONFLICT OF INTEREST}

No potential conflict of interest relevant to this article was reported.

\section{ACKNOWLEDGMENTS}

This research was supported by the Universiti Sains Malaysia under USM Short Term Grant (No. 304/PPSP/61313115).

\section{REFERENCES}

1. Ferlay J, Soerjomataram I, Ervik M, Dikshit R, Eser S, Mathers C, et al., editors. GLOBOCAN 2012: estimated cancer incidence, mortality and prevalence worldwide in 2012 v1.0. IARC CancerBase No. 11. Lyon: International Agency for Research on Cancer, 2013.

2. Pourhoseingholi MA. Increased burden of colorectal cancer in Asia. World J Gastrointest Oncol 2012;4:68-70.

3. Levin B, Lieberman DA, McFarland B, Smith RA, Brooks D, Andrews KS, et al. Screening and surveillance for the early detection of colorectal cancer and adenomatous polyps, 2008: a joint guideline from the American Cancer Society, the US Multi-Society 
Task Force on Colorectal Cancer, and the American College of Radiology. CA Cancer J Clin 2008;58:130-60.

4. Yusoff HM, Daud N, Noor NM, Rahim AA. Participation and barriers to colorectal cancer screening in Malaysia. Asian Pac J Cancer Prev 2012;13:3983-7.

5. Paspatis GA, Vardas E, Theodoropoulou A, Manolaraki MM, Charoniti I, Papanikolaou N, et al. Complications of colonoscopy in a large public county hospital in Greece. A 10-year study. Dig Liver Dis 2008;40:951-7.

6. Ayling RM. New faecal tests in gastroenterology. Ann Clin Biochem 2012;49(Pt 1):44-54.

7. Pox C. Colon cancer screening: which non-invasive filter tests? Dig Dis 2011;29 Suppl 1:56-9.

8. Malaysian Society of Gastroenterology \& Hepatology; College of Surgeons of Malaysia; Academy of Medicine, Malaysia. Screening for colorectal cancer in Malaysia: consensus/clinical practice guidelines. Kuala Lumpur: Ministry of Health, Malaysia; 2001.

9. Sung JJ, Chan FK, Leung WK, Wu JC, Lau JY, Ching J, et al. Screening for colorectal cancer in Chinese: comparison of fecal occult blood test, flexible sigmoidoscopy, and colonoscopy. Gastroenterology 2003;124:608-14.

10. Tsoi KK, Ng SS, Leung MC, Sung JJ. Cost-effectiveness analysis on screening for colorectal neoplasm and management of colorectal cancer in Asia. Aliment Pharmacol Ther 2008;28:35363.

11. Mazurek S. Pyruvate kinase M2: a key enzyme of the tumor metabolome and its medical relevance. Biomed Res 2012;23:133-41.

12. Zhou CF, Li XB, Sun H, Zhang B, Han YS, Jiang Y, et al. Pyruvate kinase type M2 is upregulated in colorectal cancer and promotes proliferation and migration of colon cancer cells. IUBMB Life 2012;64:775-82.

13. Christofk HR, Vander Heiden MG, Harris MH, Ramanathan A, Gerszten RE, Wei R, et al. The M2 splice isoform of pyruvate kinase is important for cancer metabolism and tumour growth. Nature 2008;452:230-3.

14. Hardt PD, Mazurek S, Toepler M, Schlierbach P, Bretzel RG, Eigenbrodt E, et al. Faecal tumour M2 pyruvate kinase: a new, sensitive screening tool for colorectal cancer. Br J Cancer 2004;91: 980-4.

15. Koss K, Maxton D, Jankowski JA. Faecal dimeric M2 pyruvate kinase in colorectal cancer and polyps correlates with tumour staging and surgical intervention. Colorectal Dis 2008;10:244-8.

16. Abdullah M, Rani AA, Simadibrata M, Fauzi A, Syam AF. The value of fecal tumor M2 pyruvate kinase as a diagnostic tool for colorectal cancer screening. Acta Med Indones 2012;44:94-9.

17. Hathurusinghe HR, Goonetilleke KS, Siriwardena AK. Current status of tumor M2 pyruvate kinase (tumor M2-PK) as a biomarker of gastrointestinal malignancy. Ann Surg Oncol 2007;14: 2714-20.

18. Kumar Y, Tapuria N, Kirmani N, Davidson BR. Tumour M2-pyruvate kinase: a gastrointestinal cancer marker. Eur J Gastroenterol Hepatol 2007;19:265-76.

19. McLoughlin R, Shiel E, Sebastian S, Ryan B, O’Connor HJ, O'Morain C. Tumor M2-PK, a novel screening tool for colorectal cancer. In: National Cancer Research Institute. Poster abstracts and trade exhibition book: NCRI Cancer Conference; 2005 Oct 2-5; Birmingham, UK. London: Callisto; 2005. p. 202.

20. Tonus C, Sellinger M, Koss K, Neupert G. Faecal pyruvate kinase isoenzyme type M2 for colorectal cancer screening: a meta-analysis. World J Gastroenterol 2012;18:4004-11.

21. Sithambaram S, Hilmi I, Goh KL. The diagnostic accuracy of the M2 pyruvate kinase quick stool test: a rapid office based assay test for the detection of colorectal cancer. PLoS One 2015;10:e0131616.

22. Haug U, Rothenbacher D, Wente MN, Seiler CM, Stegmaier C, Brenner H. Tumour M2-PK as a stool marker for colorectal cancer: comparative analysis in a large sample of unselected older adults vs colorectal cancer patients. Br J Cancer 2007;96:1329-34.

23. Kloer HU, Hardt PD, Schlierbach P, Toepler M. The tumor metabolic marker Tumor M2-PK in stool: a new biomarker for colorectal cancer. J Clin Oncol 2005;23(16 Suppl):3598.

24. Haug U, Hundt S, Brenner H. Sensitivity and specificity of faecal tumour M2 pyruvate kinase for detection of colorectal adenomas in a large screening study. Br J Cancer 2008;99:133-5.

25. Shastri Y, Povse N, Schröder O, Stein J. AGA Abstracts. W1141 Evaluation of a novel fecal marker- fecal tumor pyruvate kinase type M2 (M2-PK) and its comparison with calprotectin in patients with inflammatory bowel disease: a prospective multicenter study. Gastroenterology 2008;134(4 Suppl):A-642.

26. Walkowiak J, Banasiewicz T, Krokowicz P, Hansdorfer-Korzon R, Drews M, Herzig KH. Fecal pyruvate kinase (M2-PK): a new predictor for inflammation and severity of pouchitis. Scand J Gastroenterol 2005;40:1493-4.

27. Ahn SB, Han DS, Bae JH, Byun TJ, Kim JP, Eun CS. The miss rate for colorectal adenoma determined by quality-adjusted, back-toback colonoscopies. Gut Liver 2012;6:64-70. 